\title{
Energy and Mean-Payoff Parity Markov Decision Processes
}

\author{
Krishnendu Chatterjee ${ }^{1}$ and Laurent Doyen ${ }^{2}$ \\ 1 IST Austria (Institute of Science and Technology Austria) \\ ${ }^{2}$ LSV, ENS Cachan \& CNRS, France
}

\begin{abstract}
We consider Markov Decision Processes (MDPs) with mean-payoff parity and energy parity objectives. In system design, the parity objective is used to encode $\omega$-regular specifications, and the mean-payoff and energy objectives can be used to model quantitative resource constraints. The energy condition requires that the resource level never drops below 0 , and the mean-payoff condition requires that the limit-average value of the resource consumption is within a threshold. While these two (energy and mean-payoff) classical conditions are equivalent for two-player games, we show that they differ for MDPs. We show that the problem of deciding whether a state is almost-sure winning (i.e., winning with probability 1 ) in energy parity MDPs is in NP $\cap$ coNP, while for meanpayoff parity MDPs, the problem is solvable in polynomial time, improving a recent PSPACE bound.
\end{abstract}

\section{Introduction}

Markov decision processes (MDPs) are a standard model for systems that exhibit both stochastic and nondeterministic behaviour. The nondeterminism represents the freedom of choice of control actions, while the probabilities describe the uncertainty in the response of the system to control actions. The control problem for MDPs asks whether there exists a strategy (or policy) to select control actions in order to achieve a certain goal with a certain probability. MDPs have been used in several areas such as planning, probabilistic reactive programs, verification and synthesis of (concurrent) probabilistic systems $[12,24,1]$.

The control problem may specify a goal as a set of desired traces (such as $\omega$-regular specifications), or as a quantitative optimization objective for a payoff function on the traces of the MDP. Typically, discounted-payoff and mean-payoff functions have been studied [15]. Recently, the energy objectives (corresponding to total-payoff functions) have been considered in the design of resource-constrained embedded systems $[3,21,7]$ such as power-limited systems, as well as in queueing processes, and gambling models (see also [4] and references therein). The energy objective requires that the sum of the rewards be always nonnegative along a trace. Energy objective can be expressed in the setting of boundaryless one-counter MDPs [4]. In the case of MDPs, achieving energy objective with probability 1 is equivalent to achieving energy objective in the stronger setting of a two-player game where the probabilistic choices are replaced by adversarial choice. This is because if a trace $\rho$ violates the energy condition in the game, then a finite prefix of $\rho$ would have a negative energy, and this finite prefix has positive probability in 
the MDP. Note that in the case of two-player games, the energy objective is equivalent to enforce nonnegative mean-payoff value $[3,5]$.

In this paper, we consider MDPs equipped with the combination of a parity objective (which is a canonical way to express the $\omega$-regular conditions [23]), and a quantitative objective specified as either mean-payoff or energy condition. Special cases of the parity objective include reachability and fairness objectives such as Büchi and coBüchi conditions. Such combination of quantitative and qualitative objectives is crucial in the design of reactive systems with both resource constraints and functional requirements $[6,11,3$, 2]. For example, Kucera and Strazvosky consider the combination of PCTL with meanpayoff objectives for MDPs and present an EXPTIME algorithm [19]. In the case of energy parity condition, it can also be viewed as a natural extension of boundaryless one-counter MDPs with fairness conditions.

Consider the MDP in Fig. 1, with the objective to visit the Büchi state $q_{2}$ infinitely often, while maintaining the energy level (i.e., the sum of the transition weights) nonnegative. A winning strategy from $q_{0}$ would loop 20 times on $q_{0}$ to accumulate energy and then it can afford to reach the probabilistic state from which the Büchi state is reached with probability $1 / 2$ and cost 20 . If the Büchi state is not reached immediately, then the strategy needs to recharge 10 units of energy and try again. This strategy uses memory and it is also winning with probability 1 for the nonnegative mean-payoff Büchi objective. In general however, the energy and mean-payoff parity objectives do not coincide (see later the example in Fig. 2). In particular, the memory requirement for energy parity objective is finite (at most exponential) while it may be infinite for mean-payoff parity.

We study the computational complexity of the problem of deciding if there exists a strategy to achieve energy parity objective, or mean-payoff parity objective with probability 1 (i.e., almost-surely). We provide tight bounds for this problems in the following sense.

1. For energy parity MDPs, we show that the problem is in NP $\cap$ coNP, and present a pseudo-polynomial time algorithm. Our bounds are the best conceivable upper bound unless parity games can be solved in $\mathrm{P}^{3}$, which is a long-standing open question.

2. For mean-payoff parity MDPs, we show that the problem is solvable in polynomial time (and thus PTIME-complete). Our result improves the recent PSPACE upper bound of [17] for this problem.

We refer to $[12,16,10]$ for importance of the computation of almost-sure winning set related to robust solutions (independence of precise transition probabilities) and the more general quantitative problem. The computation of the almost-sure winning set in MDPs typically relies either on the end-component analysis, or analysis of attractors and sub-MDPs. The result of [17] for mean-payoff parity MDPs uses the analysis of attractors and sub-MDPs and gives a nested recursive PSPACE algorithm for the problem similar to the classical algorithm for parity games. Our results rely on the end-component analysis, but in a much more refined way than the standard analysis, to obtain a polynomial-time algorithm. Our proof combines techniques for mean-payoff

\footnotetext{
${ }^{3}$ Parity games polynomially reduce to two-player energy games [18,3,5], and thus to energy MDPs. Hence the problem for almost-sure energy parity MDPs is at least as hard as solving two player parity games.
} 


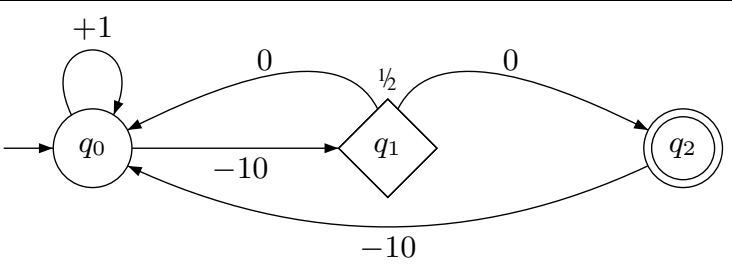

Fig. 1. An energy Büchi MDP. The player- 1 states are $q_{0}, q_{2}$, and the probabilistic state is $q_{1}$.

and parity objectives to produce infinite-memory strategy witnesses, which is necessary in general. We present an algorithm that iterates successively over even priorities $2 i$ and computes almost-sure winning end-components with the even priority $2 i$ as the best priority.

For energy parity MDPs the end-component based analysis towards polynomialtime algorithm does not work since solving energy parity MDPs is at least as hard as solving two-player parity games. Instead, for energy parity MDPs, we present a quadratic reduction to two-player energy Büchi games which are in NP $\cap$ coNP and solvable in pseudo-polynomial time [7].

Due to lack of space we present the detailed proofs in the appendix.

\section{Definitions}

Probability distributions. A probability distribution over a finite set $A$ is a function $\kappa: A \rightarrow[0,1]$ such that $\sum_{a \in A} \kappa(a)=1$. The support of $\kappa$ is the set $\operatorname{Supp}(\kappa)=\{a \in$ $A \mid \kappa(a)>0\}$. We denote by $\mathcal{D}(A)$ the set of probability distributions on $A$.

Markov Decision Processes. A Markov Decision Process (MDP) $M=(Q, E, \delta)$ consists of a finite set $Q$ of states partitioned into player-1 states $Q_{1}$ and probabilistic states $Q_{P}$ (i.e., $Q=Q_{1} \cup Q_{P}$ ), a set $E \subseteq Q \times Q$ of edges such that for all $q \in Q$, there exists (at least one) $q^{\prime} \in Q$ such that $\left(q, q^{\prime}\right) \in E$, and a probabilistic transition function $\delta: Q_{P} \rightarrow \mathcal{D}(Q)$ such that for all $q \in Q_{P}$ and $q^{\prime} \in Q$, we have $\left(q, q^{\prime}\right) \in E$ iff $\delta(q)\left(q^{\prime}\right)>0$. We often write $\delta\left(q, q^{\prime}\right)$ for $\delta(q)\left(q^{\prime}\right)$. For a state $q \in Q$, we denote by $E(q)=\left\{q^{\prime} \in Q \mid\left(q, q^{\prime}\right) \in E\right\}$ the set of possible successors of $q$.

End-components and Markov chains. A set $U \subseteq Q$ is $\delta$-closed if for all $q \in U \cap Q_{P}$ we have $\operatorname{Supp}(\delta(q)) \subseteq U$. The sub-MDP induced by a $\delta$-closed set $U$ is $M \uparrow U=$ $(U, E \cap(U \times U), \delta)$. Note that $M \uparrow U$ is an MDP if for all $q \in U$ there exists $q^{\prime} \in U$ such that $\left(q, q^{\prime}\right) \in E$. A Markov chain is a special case of MDP where $Q_{1}=\emptyset$. A closed recurrent set for a Markov chain is a $\delta$-closed set $U \subseteq Q$ which is strongly connected. End-components in MDPs play a role equivalent to closed recurrent sets in Markov chains. Given an MDP $M=(Q, E, \delta)$ with partition $\left(Q_{1}, Q_{P}\right)$, a set $U \subseteq Q$ of states is an end-component if $U$ is $\delta$-closed and the sub-MDP $M \uparrow U$ is strongly connected $[12,13]$. We denote by $\mathcal{E}(M)$ the set of end-components of an MDP $M$.

Plays. An MDP can be viewed as the arena of a game played for infinitely many rounds from a state $q_{0} \in Q$ as follows. If the game is in a player- 1 state $q$, then player 1 chooses the successor state in the set $E(q)$; otherwise the game is in a probabilistic state $q$, and the successor is chosen according to the probability distribution $\delta(q)$. This game results 
in a play from $q_{0}$, i.e., an infinite path $\rho=q_{0} q_{1} \ldots$ such that $\left(q_{i}, q_{i+1}\right) \in E$ for all $i \geq 0$. The prefix of length $n$ of $\rho$ is denoted by $\rho(n)=q_{0} \ldots q_{n}$, the last state of $\rho(n)$ is $\operatorname{Last}(\rho(n))=q_{n}$. We write $\Omega$ for the set of all plays.

Strategies. A strategy (for player 1) is a function $\sigma: Q^{*} Q_{1} \rightarrow \mathcal{D}(Q)$ such that for all $\rho \in Q^{*}, q \in Q_{1}$, and $q^{\prime} \in Q_{P}$, if $\sigma(\rho \cdot q)\left(q^{\prime}\right)>0$, then $\left(q, q^{\prime}\right) \in E$. We denote by $\Sigma$ the set of all strategies. An outcome of $\sigma$ from $q_{0}$ is a play $q_{0} q_{1} \ldots$ where $q_{i+1} \in$ $\operatorname{Supp}\left(\sigma\left(q_{0} \ldots q_{i}\right)\right)$ for all $i \geq 0$ such that $q_{i} \in Q_{1}$.

Outcomes and measures. Once a starting state $q \in Q$ and a strategy $\sigma \in \Sigma$ are fixed, the outcome of the game is a random walk $\omega_{q}^{\sigma}$ for which the probabilities of every event $\mathcal{A} \subseteq \Omega$, which is a measurable set of plays, are uniquely defined [24]. For a state $q \in Q$ and an event $\mathcal{A} \subseteq \Omega$, we denote by $\mathbb{P}_{q}^{\sigma}(\mathcal{A})$ the probability that a play belongs to $\mathcal{A}$ if the game starts from the state $q$ and player 1 follows the strategy $\sigma$. For a measurable function $f: \Omega \rightarrow \mathbb{R}$ we denote by $\mathbb{E}_{q}^{\sigma}[f]$ the expectation of the function $f$ under the probability measure $\mathbb{P}_{q}^{\sigma}(\cdot)$.

Strategies that do not use randomization are called pure. A player-1 strategy $\sigma$ is pure if for all $\rho \in Q^{*}$ and $q \in Q_{1}$, there is a state $q^{\prime} \in Q$ such that $\sigma(\rho \cdot q)\left(q^{\prime}\right)=1$.

Finite-memory strategies. A strategy uses finite-memory if it can be encoded by a deterministic transducer $\left\langle\mathrm{Mem}, m_{0}, \alpha_{u}, \alpha_{n}\right\rangle$ where Mem is a finite set (the memory of the strategy), $m_{0} \in$ Mem is the initial memory value, $\alpha_{u}:$ Mem $\times Q \rightarrow$ Mem is an update function, and $\alpha_{n}: \operatorname{Mem} \times Q_{1} \rightarrow \mathcal{D}(Q)$ is a next-move function. The size of the strategy is the number $|\mathrm{Mem}|$ of memory values. If the game is in a player-1 state $q$, and $m$ is the current memory value, then the strategy chooses the next state $q^{\prime}$ according to the probability distribution $\alpha_{n}(m, q)$, and the memory is updated to $\alpha_{u}(m, q)$. Formally, $\left\langle\right.$ Mem, $\left.m_{0}, \alpha_{u}, \alpha_{n}\right\rangle$ defines the strategy $\sigma$ such that $\sigma(\rho \cdot q)=$ $\alpha_{n}\left(\hat{\alpha}_{u}\left(m_{0}, \rho\right), q\right)$ for all $\rho \in Q^{*}$ and $q \in Q_{1}$, where $\hat{\alpha}_{u}$ extends $\alpha_{u}$ to sequences of states as expected. A strategy is memoryless if $|\mathrm{Mem}|=1$. For a finite-memory strategy $\sigma$, let $M_{\sigma}$ be the Markov chain obtained as the product of $M$ with the transducer defining $\sigma$, where $\left(\langle m, q\rangle,\left\langle m^{\prime}, q^{\prime}\right\rangle\right)$ is an edge in $M_{\sigma}$ if $m^{\prime}=\alpha_{u}(m, q)$ and either $q \in Q_{1}$ and $q^{\prime} \in \operatorname{Supp}\left(\alpha_{n}(m, q)\right)$, or $q \in Q_{P}$ and $\left(q, q^{\prime}\right) \in E$.

Two-player games. A two-player game is a graph $G=(Q, E)$ with the same assumptions as for MDP, except that the partition of $Q$ is denoted $\left(Q_{1}, Q_{2}\right)$ where $Q_{2}$ is the set of player-2 states. The notions of play, strategies (in particular strategies for player 2), and outcome are analogous to the case of MDP [7].

Objectives. An objective for an MDP $M$ (or game $G$ ) is a set $\phi \subseteq \Omega$ of infinite paths. Let $p: Q \rightarrow \mathbb{N}$ be a priority function and $w: E \rightarrow \mathbb{Z}$ be a weight function ${ }^{4}$ where positive numbers represent rewards. We denote by $W$ the largest weight (in absolute value) according to $w$. The energy level of a prefix $\gamma=q_{0} q_{1} \ldots q_{n}$ of a play is $\operatorname{EL}(w, \gamma)=\sum_{i=0}^{n-1} w\left(q_{i}, q_{i+1}\right)$, and the mean-payoff value $^{5}$ of a play $\rho=q_{0} q_{1} \ldots$ is $\operatorname{MP}(w, \rho)=\liminf _{n \rightarrow \infty} \frac{1}{n} \cdot \operatorname{EL}(w, \rho(n))$. In the sequel, when the weight function $w$ is clear from the context we omit it and simply write $\operatorname{EL}(\gamma)$ and $\operatorname{MP}(\rho)$. We denote by

\footnotetext{
${ }^{4}$ Sometimes we take the freedom to use rational weights (i.e., $w: E \rightarrow \mathbb{Q}$ ), while we always assume that weights are integers encoded in binary for complexity results.

${ }^{5}$ The results of this paper hold for the definition of mean-payoff value using lim sup instead of $\lim$ inf.
} 
$\operatorname{lnf}(\rho)$ the set of states that occur infinitely often in $\rho$, and we consider the following objectives:

- Parity objectives. The parity objective $\operatorname{Parity}(p)=\{\rho \in \Omega \mid \min \{p(q) \mid q \in$ $\operatorname{Inf}(\rho)\}$ is even $\}$ requires that the minimum priority visited infinitely often be even. The special cases of Büchi and coBüchi objectives correspond to the case with two priorities, $p: Q \rightarrow\{0,1\}$ and $p: Q \rightarrow\{1,2\}$ respectively.

- Energy objectives. Given an initial credit $c_{0} \in \mathbb{N}$, the energy objective $\operatorname{PosEnergy}\left(c_{0}\right)=\left\{\rho \in \Omega \mid \forall n \geq 0: c_{0}+\operatorname{EL}(\rho(n)) \geq 0\right\}$ requires that the energy level be always positive.

- Mean-payoff objectives. Given a threshold $\nu \in \mathbb{Q}$, the mean-payoff objective MeanPayoff $^{\geq \nu}=\{\rho \in \Omega \mid \operatorname{MP}(\rho) \geq \nu\}$ (resp. MeanPayoff ${ }^{>\nu}=\{\rho \in \Omega \mid$ $\operatorname{MP}(\rho)>\nu\}$ ) requires that the mean-payoff value be at least $\nu$ (resp. strictly greater than $\nu$ ).

- Combined objectives. The energy parity objective Parity $(p) \cap \operatorname{PosEnergy}\left(c_{0}\right)$ and the mean-payoff parity objective Parity $(p) \cap$ MeanPayoff $^{\sim \nu}$ (for $\sim \in\{\geq,>\}$ ) combine the requirements of parity and energy (resp., mean-payoff) objectives.

Almost-sure winning strategies. For MDPs, we say that a player- 1 strategy $\sigma$ is almost-sure winning in a state $q$ for an objective $\phi$ if $\mathbb{P}_{q}^{\sigma}(\phi)=1$. For two-player games, we say that a player-1 strategy $\sigma$ is winning in a state $q$ for an objective $\phi$ if all outcomes of $\sigma$ starting in $q$ belong to $\phi$. For energy objectives with unspecified initial credit, we also say that a strategy is (almost-sure) winning if it is (almost-sure) winning for some finite initial credit.

Decision problems. We are interested in the following problems. Given an MDP $M$ with weight function $w$ and priority function $p$, and a state $q_{0}$,

- the energy parity problem asks whether there exists a finite initial credit $c_{0} \in \mathbb{N}$ and an almost-sure winning strategy for the energy parity objective from $q_{0}$ with initial credit $c_{0}$. We are also interested in computing the minimum initial credit in $q_{0}$ which is the least value of initial credit for which there exists an almost-sure winning strategy for player 1 in $q_{0}$. A strategy for player 1 is optimal in $q_{0}$ if it is winning from $q_{0}$ with the minimum initial credit;

- the mean-payoff parity problem asks whether there exists an almost-sure winning strategy for the mean-payoff parity objective with threshold 0 from $q_{0}$. Note that it is not restrictive to consider mean-payoff objectives with threshold 0 because for $\sim \in\{\geq,>\}$, we have $\operatorname{MP}(w, \rho) \sim \nu$ iff $\operatorname{MP}(w-\nu, \rho) \sim 0$, where $w-\nu$ is the weight function that assigns $w(e)-\nu$ to each edge $e \in E$.

The two-player game versions of these problems are defined analogously [7]. It is known that the initial credit problem for simple two-player energy games [6,3], as well as for two-player parity games [14] can be solved in NP $\cap$ coNP because memoryless strategies are sufficient to win. Moreover, parity games reduce in polynomial time to mean-payoff games [18], which are log-space equivalent to energy games [3,5]. It is a long-standing open question to know if a polynomial-time algorithm exists for these problems. Finally, energy parity games and mean-payoff parity games are solvable in $\mathrm{NP} \cap$ coNP although winning strategies may require exponential and infinite memory respectively, even in one-player games (and thus also in MDPs) [11,7].

The decision problem for MDPs with parity objective, as well as with mean-payoff objective, can be solved in polynomial time $[15,12,10,13]$. However, the problem is in NP $\cap$ coNP for MDPs with energy objective because an MDP with energy objective 


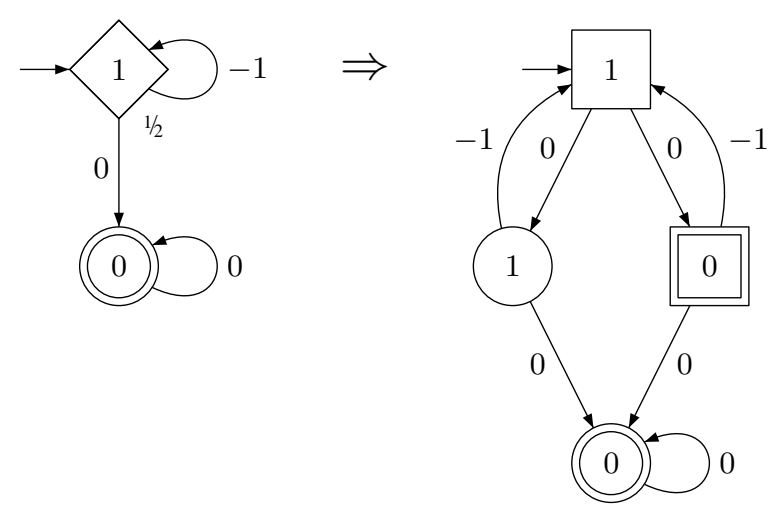

Fig. 2. The gadget construction is wrong for mean-payoff parity MDPs. Player 1 is almost-sure winning for mean-payoff Büchi in the MDP (on the left) but player 1 is losing in the two-player game (on the right) because player 2 (box-player) can force a negative-energy cycle.

is equivalent to a two-player energy game (where the probabilistic states are controlled by player 2). Indeed (1) a winning strategy in the game is trivially almost-sure winning in the MDP, and (2) if an almost-sure winning strategy $\sigma$ in the MDP was not winning in the game, then for all initial credit $c_{0}$ there would exist an outcome $\rho$ of $\sigma$ such that $c_{0}+\operatorname{EL}(\rho(i))<0$ for some position $i \geq 0$. The prefix $\rho(i)$ has a positive probability in the MDP, in contradiction with the fact that $\sigma$ is almost-sure winning. As a consequence, solving MDP with energy objectives is at least as hard as solving parity games.

In this paper, we show that the decision problem for MDPs with energy parity objective is in NP $\cap$ coNP, which is the best conceivable upper bound unless parity games can be solved in P. And for MDPs with mean-payoff parity objective, we show that the decision problem can be solved in polynomial time, improving a recent PSPACE bound [17].

The MDP in Fig. 2 on the left, which is essentially a Markov chain, is an example where the mean-payoff parity condition is satisfied almost-surely, while the energy parity condition is not, no matter the value of the initial credit. For initial credit $c_{0}$, the energy will drop below 0 with positive probability, namely $\frac{1}{2^{c_{0}+1}}$.

End-component lemma. We now present an important lemma about end-components from $[12,13]$ that we use in the proofs of our result. It states that for arbitrary strategies (memoryless or not), with probability 1 the set of states visited infinitely often along a play is an end-component. This lemma allows us to derive conclusions on the (infinite) set of plays in an MDP by analyzing the (finite) set of end-components in the MDP.

Lemma 1. [12, 13] Given an MDP $M$, for all states $q \in Q$ and all strategies $\sigma \in \Sigma$, we have $\mathbb{P}_{q}^{\sigma}(\{\omega \mid \operatorname{lnf}(\omega) \in \mathcal{E}(M)\})=1$.

\section{MDPs with Energy Parity Objectives}

We show that energy parity MDPs can be solved in NP $\cap$ coNP, using a reduction to two-player energy Büchi games. Our reduction also preserves the value of the minimum 

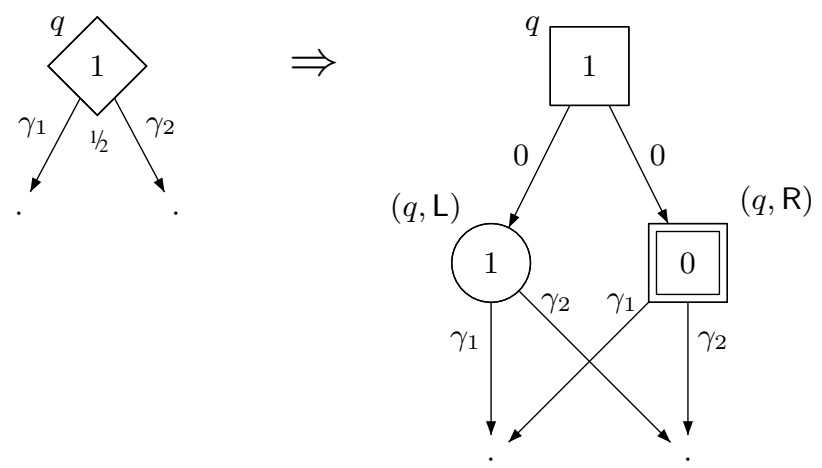

Fig. 3. Gadget for probabilistic states in energy Büchi MDP. Diamonds are probabilistic states, circles are player 1 states, and boxes are player 2 states.

initial credit. Therefore, we obtain a pseudo-polynomial algorithm for this problem, which also computes the minimum initial credit. Moreover, we show that the memory requirement for almost-sure winning strategies is at most $2 \cdot|Q| \cdot W$, which is essentially optimal $^{6}$.

We first establish the results for the special case of energy Büchi MDPs. We present a reduction of the energy Büchi problem for MDPs to the energy Büchi problem for two-player games. The result then follows from the fact that the latter problem is in $\mathrm{NP} \cap$ coNP and solvable in pseudo-polynomial time [7].

Given an MDP $M$, we can assume without loss of generality that every probabilistic state has priority 1 , and has two outgoing transitions with probability $1 / 2$ each $[25$, Section 6]. We construct a two-player game $G$ by replacing every probabilistic state of $M$ by a gadget as in Fig. 3. The probabilistic states $q$ of $M$ are mapped to player-2 states in $G$ with two successors $(q, \mathrm{~L})$ and $(q, \mathrm{R})$. Intuitively, player 2 chooses $(q, \mathrm{~L})$ to check whether player 1 can enforce the Büchi condition almost-surely. This is the case if player 1 can reach a Büchi state (with priority 0 ) infinitely often when he controls the probabilistic states (otherwise, no Büchi state is ever visited, and since $(\cdot, L)$ states have priority 1, the Büchi condition is not realized in $G)$. And player 2 chooses $(q, \mathrm{R})$ to check that the energy condition is satisfied. If player 2 can exhaust the energy level in $G$, then the corresponding play prefix has positive probability in $M$. Note that $(q, \mathrm{R})$ has priority 0 and thus cannot be used by player 2 to spoil the Büchi condition.

Formally, given $M=(Q, E, \delta)$ with partition $\left(Q_{1}, Q_{P}\right)$ of $Q$, we construct a game $G=\left(Q^{\prime}, E^{\prime}\right)$ with partition $\left(Q_{1}^{\prime}, Q_{P}^{\prime}\right)$ where $Q_{1}^{\prime}=Q_{1} \cup\left(Q_{P} \times\{\mathrm{L}\}\right)$ and $Q_{2}^{\prime}=$ $Q_{P} \cup\left(Q_{P} \times\{\mathrm{R}\}\right)$, see also Fig. 3. The states in $Q^{\prime}$ that are already in $Q$ get the same priority as in $M$, the states $(\cdot, \mathrm{L})$ have priority 1 , and the states $(\cdot, \mathrm{R})$ have priority 0 . The set $E^{\prime}$ contains the following edges:

- all edges $\left(q, q^{\prime}\right) \in E$ such that $q \in Q_{1}$;

- edges $(q,(q, d)),\left((q, d), q^{\prime}\right)$ for all $q \in Q_{P}, d \in\{\mathrm{L}, \mathrm{R}\}$, and $q^{\prime} \in \operatorname{Supp}(\delta(q))$.

\footnotetext{
${ }^{6}$ Example 1 in [7] shows that memory of size $2 \cdot(|Q|-1) \cdot W+1$ may be necessary.
} 
The edges $\left(q, q^{\prime}\right)$ and $\left((q, d), q^{\prime}\right)$ in $E^{\prime}$ get the same weight as $\left(q, q^{\prime}\right)$ in $M$, and all edges $(q,(q, d))$ get weight 0 .

Lemma 2. Given an MDP $M$ with energy Büchi objective, we can construct in linear time a two-player game $G$ with energy Büchi objective such that for all states $q_{0}$ in $M$, there exists an almost-sure winning strategy from $q_{0}$ in $M$ if and only if there exists a winning strategy from $q_{0}$ in $G$ (with the same initial credit).

Note that the reduction presented in the proof of Lemma 2 would not work for mean-payoff Büchi MDPs. Consider the MDP on Fig. 2 for which the gadget-based reduction to two-player games is shown on the right. The game is losing for player 1 both for energy and mean-payoff parity, simply because player 2 can always choose to loop through the box states, thus realizing a negative energy and mean-payoff value (no matter the initial credit). However player 1 is almost-sure winning in the mean-payoff parity MDP (on the left in Fig. 2).

While the reduction in the proof of Lemma 2 gives a game with $n^{\prime}=\left|Q_{1}\right|+3$. $\left|Q_{P}\right|$ states, the structure of the gadgets (see Fig. 3) is such that the energy level is independent of which of the transitions $(q,(q, \mathrm{~L}))$ or $(q,(q, \mathrm{R}))$ is taken. Since from the result of [7, Lemma 8] and its proof, it follows that the memory updates in winning strategies for energy Büchi games can be done according to the energy level of the play prefix, it follows that the memory bound of $2 \cdot n \cdot W$ can be transfered to almost-sure winning strategies in Energy Büchi MDPs, where $n=\mid$ Win $\cap Q_{1} \mid$ is the number of player 1 almost-sure winning states. Also, the pseudo-polynomial algorithm for solving two-player energy Büchi games can be used for MDPs, with the same $O\left(|E| \cdot|Q|^{5} \cdot W\right)$ complexity [7, Table 1] .

Using Lemma 2, we solve energy parity MDPs by a reduction to energy Büchi MDPs. The key idea of the reduction is that if player 1 has an almost-sure winning strategy for the energy parity objective, then player 1 can choose an even priority $2 i$ and decide to satisfy the energy objective along with satisfying that priority $2 i$ is visited infinitely often, and priorities less than $2 i$ are visited finitely often.

W.l.o.g. we assume that player- 1 states and probabilistic states alternate, i.e. $E(q) \subseteq$ $Q_{1}$ for all $q \in Q_{P}$, and $E(q) \subseteq Q_{P}$ for all $q \in Q_{1}$. The reduction is then as follows. Given an MDP $M=(Q, E, \delta)$ with a priority function $p: Q \rightarrow \mathbb{N}$ and a weight function $w: E \rightarrow \mathbb{Z}$, we construct $\left\langle M^{\prime}, p^{\prime}, w^{\prime}\right\rangle$ as follows. $M^{\prime}$ is the MDP $M=$ $\left(Q^{\prime}, E^{\prime}, \delta^{\prime}\right)$ where:

- $Q^{\prime}=Q \cup(Q \times\{0,2, \ldots, 2 r\}) \cup\{$ sink $\}$ where $2 r$ is the largest even priority of a state in $Q$. Intuitively, a state $(q, i) \in Q^{\prime}$ corresponds to the state $q$ of $M$ from which player 1 will ensure to visit priority $i$ (which is even) infinitely often, and never visit priority smaller than $i$;

- $E^{\prime}$ contains $E \cup\{($ sink, sink $)\}$ and the following edges. For each probabilistic state $q \in Q_{P}$, for $i=0,2, \ldots, 2 r$,

- $(a)$ if $p\left(q^{\prime}\right) \geq i$ for all $q^{\prime} \in E(q)$, then $\left((q, i),\left(q^{\prime}, i\right)\right) \in E^{\prime}$ for all $q^{\prime} \in E(q)$,

- $(b)$ otherwise, $((q, i)$, sink $) \in E^{\prime}$.

For each player 1 state $q \in Q_{1}$, for each $q^{\prime} \in E(q)$, for $i=0,2, \ldots, 2 r$,

- (a) $(q$, sink $) \in E^{\prime}$ and $((q, i)$, sink $) \in E^{\prime}$, and

- (b) if $p\left(q^{\prime}\right) \geq i$, then $\left(q,\left(q^{\prime}, i\right)\right) \in E^{\prime}$ and $\left((q, i),\left(q^{\prime}, i\right)\right) \in E^{\prime}$. 
The partition $\left(Q_{1}^{\prime}, Q_{P}^{\prime}\right)$ of $Q^{\prime}$ is defined by $Q_{1}^{\prime}=Q_{1} \cup\left(Q_{1} \times\{0,2, \ldots, 2 r\}\right) \cup\{$ sink $\}$ and $Q_{P}^{\prime}=Q^{\prime} \backslash Q_{1}^{\prime}$. The weight of the edges $\left(q, q^{\prime}\right),\left(q,\left(q^{\prime}, i\right)\right)$ and $\left((q, i),\left(q^{\prime}, i\right)\right)$ according to $w^{\prime}$ is the same as the weight of $\left(q, q^{\prime}\right)$ according to $w$. The states $(q, i)$ such that $p(q)=i$ have priority 0 according to $p^{\prime}$ (they are the Büchi states), and all the other states in $Q^{\prime}$ (including sink) have priority 1 .

Lemma 3. Given an MDP $M$ with energy parity objective, we can construct in quadratic time an MDP $M^{\prime}$ with energy Büchi objective such that for all states $q_{0}$ in $M$, there exists an almost-sure winning strategy from $q_{0}$ in $M$ if and only if there exists an almost-sure winning strategy from $q_{0}$ in $M^{\prime}$ (with the same initial credit).

From the proof of Lemma 3, it follows that the memory requirement is the same as for energy Büchi MDPs. And if the weights are in $\{-1,0,1\}$, it follows that the energy parity problem can be solved in polynomial time.

Theorem 1. For energy parity MDPs,

1. the decision problem of whether a given state is almost-sure winning is in $N P \cap$ coNP, and there is a pseudo-polynomial time algorithm in $O\left(|E| \cdot d \cdot|Q|^{5} \cdot W\right)$ to solve it;

2. memory of size $2 \cdot|Q| \cdot W$ is sufficient for almost-sure winning strategies.

\section{MDPs with Mean-payoff Parity Objectives}

In this section we present a polynomial-time algorithm for solving MDPs with meanpayoff parity objective. We first recall some useful properties of MDPs.

For an end-component $U \in \mathcal{E}(M)$, consider the memoryless strategy $\sigma_{U}$ that plays in every state $s \in U \cap Q_{1}$ all edges in $E(s) \cap U$ uniformly at random. Given the strategy $\sigma_{U}$, the end-component $U$ is a closed connected recurrent set in the Markov chain obtained by fixing $\sigma_{U}$.

Lemma 4. Given an MDP $M$ and an end-component $U \in \mathcal{E}(M)$, the strategy $\sigma_{U}$ ensures that for all states $s \in U$, we have $\mathbb{P}_{s}^{\sigma_{U}}(\{\omega \mid \operatorname{lnf}(\omega)=U\})=1$.

Expected mean-payoff value. Given an MDP $M$ with a weight function $w$, the expected mean-payoff value, denoted $\operatorname{VaIMP}(w)$, is the function that assigns to every state the maximal expectation of the mean-payoff objective that can be guaranteed by any strategy. Formally, for $q \in Q$ we have $\operatorname{ValMP}(w)(q)=\sup _{\sigma \in \Sigma} \mathbb{E}_{q}^{\sigma}(\mathrm{MP}(w))$, where $\operatorname{MP}(w)$ is the measurable function that assigns to a play $\rho$ the long-run average $\operatorname{MP}(w, \rho)$ of the weights. By the classical results of MDPs with mean-payoff objectives, it follows that there exists pure memoryless optimal strategies [15], i.e., there exists a pure memoryless optimal strategy $\sigma^{*}$ such that for all $q \in Q$ we have $\operatorname{ValMP}(w)(q)=\mathbb{E}_{q}^{\sigma^{*}}(\operatorname{MP}(w))$.

It follows from Lemma 4 that the strategy $\sigma_{U}$ ensures that from any starting state $s$, any other state $t$ is reached in finite time with probability 1 . Therefore, the value for mean-payoff parity objectives in MDPs can be obtained by computing values for endcomponents and then playing a strategy to maximize the expectation to reach the values of the end-components. 
We now present the key lemma where we show that for an MDP that is an endcomponent such that the minimum priority is even, the mean-payoff parity objective $\operatorname{Parity}(p) \cap$ MeanPayoff $\geq \nu$ is satisfied with probability 1 if the expected mean-payoff value is at least $\nu$ at some state (the result also holds for strict inequality). In other words, from the expected mean-payoff value of at least $\nu$ we ensure that both the meanpayoff and parity objective is satisfied with probability 1 from all states. The proof of the lemma considers two pure memoryless strategies: one for stochastic shortest path and the other for optimal expected mean-payoff value, and combines them to obtain an almost-sure winning strategy for the mean-payoff parity objective (details in appendix).

Lemma 5. Consider an MDP $M$ with state space $Q$, a priority function $p$, and weight function $w$ such that (a) $M$ is an end-component (i.e., $Q$ is an end-component) and (b) the smallest priority in $Q$ is even. If there is a state $q \in Q$ such that $\operatorname{ValMP}(w) \geq \nu$ (resp. $\operatorname{ValMP}(w)>\nu)$, then there exists a strategy $\sigma^{*}$ such that for all states $q \in Q$ we have $\mathbb{P}_{q}^{\sigma^{*}}\left(\operatorname{Parity}(p) \cap\right.$ MeanPayoff $\left.{ }^{\geq \nu}\right)=1\left(\right.$ resp. $\mathbb{P}_{q}^{\sigma^{*}}\left(\right.$ Parity $(p) \cap$ MeanPayoff $\left.\left.{ }^{>\nu}\right)=1\right)$.

Memory required by strategies. Lemma 5 shows that if the smallest priority in an endcomponent is even, then considering the sub-game restricted to the end-component, the mean-payoff parity objective is satisfied if and only if the mean-payoff objective is satisfied. The strategy constructed in Lemma 5 requires infinite memory, and in the case of loose inequality (i.e., MeanPayoff ${ }^{\nu}$ ) infinite memory is required in general (see [11] for an example on graphs), and if the inequality is strict (i.e., MeanPayoff ${ }^{>\nu}$ ), then finite memory strategies exist [17]. For the purpose of computation we show that both strict and non-strict inequality can be solved in polynomial time. Since Lemma 5 holds for both strict and non-strict inequality, in sequel of this section we consider nonstrict inequality and all the results hold for non-strict inequality as well.

Winning end-component. Given an MDP $M$ with a parity objective Parity $(p)$ and a mean-payoff objective MeanPayoff $\geq \nu$ for a weight function $w$, we call an end-component $U$ winning if (a) $\min (p(U))$ is even; and (b) there exists a state with expected mean-payoff value at least $\nu$ in the sub-MDP induced by $U$, i.e., $\max _{q \in U} \operatorname{ValMP}(w)(q) \geq \nu$ in the sub-MDP induced by $U$. We denote by $\mathcal{W}$ the set of winning end-components, and let Win $=\bigcup_{U \in \mathcal{W}} U$ be the union of the winning end-components.

Reduction to reachability of winning end-component. By Lemma 5 it follows that in every winning end-component the mean-payoff parity objective is satisfied with probability 1 . Conversely, consider an end-component $U$ that is not winning, then either the smallest priority is odd, or the maximal expected mean-payoff value that can be ensured for any state in $U$ by staying in $U$ is less than $\nu$. Hence if only states in $U$ are visited infinitely often, then with probability 1 (i) either the parity objective is not satisfied, or (ii) the mean-payoff objective is not satisfied. In other words, if an endcomponent that is not winning is visited infinitely often, then the mean-payoff parity objective is satisfied with probability 0 . It follows that the value function for MDPs with mean-payoff parity objective can be computed by computing the value function for reachability to the set Win, i.e., formally, $\sup _{\sigma \in \Sigma} \mathbb{P}_{q}^{\sigma}(\operatorname{Parity}(p) \cap$ MeanPayoff $\geq \nu)=$ $\sup _{\sigma \in \Sigma} \mathbb{P}_{q}^{\sigma}(\operatorname{Reach}($ Win) $)$, where Reach(Win) is the set of paths that reaches a state in Win at least once. Since the value function in MDPs with reachability objectives can be computed in polynomial time using linear programming [15], it suffices to present a 
polynomial-time algorithm to compute Win in order to obtain a polynomial-time algorithm for MDPs with mean-payoff parity objectives.

Computing winning end-components. The computation of the winning endcomponents is done iteratively by computing winning end-components with smallest priority 0 , then winning end-components with smallest priority 2 , and so on. The computation of Win is as follows:

- For $i \geq 0$, let $\mathcal{W}_{2 i}$ be the set of maximal end-components $U$ with states with priority at least $2 i$ and that contain at least one state with priority $2 i$, i.e., $U$ contains only states with priority at least $2 i$, and contains at least one state with priority $2 i$. Let $\mathcal{W}_{2 i}^{\prime} \subseteq \mathcal{W}_{2 i}$ be the set of maximal end-components $U \in \mathcal{W}_{2 i}$ such that there is a state $q \in U$ such that the expected mean-payoff value in the sub-MDP restricted to $U$ is at least $\nu$. Let $\mathrm{Win}_{2 i}=\bigcup_{U \in \mathcal{W}_{2 i}^{\prime}} U$.

The set Win $=\bigcup_{i=0}^{\lfloor d / 2\rfloor} \mathrm{Win}_{2 i}$ is the union of the states of the winning end-components (formal pseudo-code in the appendix).

Complexity of computing winning end-components. The winning end-component algorithm runs for $O(d)$ iterations and in each iteration requires to compute a maximal end-component decomposition and compute mean-payoff values of at most $n$ endcomponents, where $n$ is the number of states of the MDP. The maximal end-component decomposition can be achieved in polynomial time $[12,13,10]$. The mean-payoff value function of an MDP can also be computed in polynomial time using linear programming [15]. It follows that the value function of an MDP with mean-payoff parity objectives can be computed in polynomial time. The almost-sure winning set is obtained by computing almost-sure reachability to Win in polynomial time $[12,13,10]$. This polynomial-time complexity provides a tight upper bound for the problem, and closes the gap left by the PSPACE upper bound of [17].

Theorem 2. The following assertions hold:

1. The set of almost-sure winning states for mean-payoff parity objectives can be computed in polynomial time for MDPs.

2. For mean-payoff parity objectives, almost-sure winning strategies require infinite memory in general for non-strict inequality (i.e, for mean-payoff parity objectives $\operatorname{Parity}(p) \cap$ MeanPayoff $\geq \nu$ ) and finite-memory almost-sure winning strategies exist for strict inequality (i.e., for $\operatorname{Parity}(p) \cap$ MeanPayoff $^{>\nu}$ ).

Discussion. We considered MDPs with conjunction of mean-payoff parity and energy parity objectives, and presented tight complexity bounds, algorithms, and bounds for the memory required by strategies. The disjunction of mean-payoff parity and energy parity objectives are straightforward and summarized in Theorem 3 (details in appendix).

Theorem 3. The following assertions hold:

1. The set of almost-sure winning states for disjunction of mean-payoff and parity objectives can be computed in polynomial time for MDPs.

2. The decision problem of whether a given state is almost-sure winning for disjunction of energy and parity objectives is in NP $\cap$ coNP for MDPs.

\section{References}

1. A. Bianco and L. de Alfaro. Model checking of probabilistic and nondeterministic systems. In FSTTCS 95, volume 1026 of LNCS, pages 499-513, 1995. 
2. R. Bloem, K. Chatterjee, T. A. Henzinger, and B. Jobstmann. Better quality in synthesis through quantitative objectives. In Proc. of CAV, LNCS 5643, pages 140-156. Springer, 2009.

3. P. Bouyer, U. Fahrenberg, K. G. Larsen, N. Markey, and J. Srba. Infinite runs in weighted timed automata with energy constraints. In Proc. of FORMATS, LNCS 5215, pages 33-47. Springer, 2008.

4. Tomás Brázdil, Václav Brozek, Kousha Etessami, Antonín Kucera, and Dominik Wojtczak. One-counter Markov decision processes. In Proc. of SODA, pages 863-874. SIAM, 2010.

5. L. Brim, J. Chaloupka, L. Doyen, R. Gentilini, and J.-F. Raskin. Faster algorithms for meanpayoff games. Formal Methods in System Design, 2010.

6. A. Chakrabarti, L. de Alfaro, T. A. Henzinger, and M. Stoelinga. Resource interfaces. In Proc. of EMSOFT, LNCS 2855, pages 117-133. Springer, 2003.

7. K. Chatterjee and L. Doyen. Energy parity games. In Proc. of ICALP: Automata, Languages and Programming (Part II), LNCS 6199, pages 599-610. Springer, 2010.

8. K. Chatterjee and L. Doyen. Energy and mean-payoff parity Markov decision processes. Technical report, IST Austria, Feb, 2011. http://pub.ist.ac.at/Pubs/ TechRpts/2011/IST-2011-0001.pdf.

9. K. Chatterjee, L. Doyen, H. Gimbert, and T. A. Henzinger. Randomness for free. In Proc. of MFCS, LNCS 6281, pages 246-257. Springer-Verlag, 2010.

10. K. Chatterjee and M. Henzinger. Faster and dynamic algorithms for maximal end-component decomposition and related graph problems in probabilistic verification. In Proc. of SODA. ACM SIAM, 2011.

11. K. Chatterjee, T. A. Henzinger, and M. Jurdziński. Mean-payoff parity games. In Proc. of LICS, pages 178-187. IEEE Computer Society, 2005.

12. C. Courcoubetis and M. Yannakakis. The complexity of probabilistic verification. J. ACM, 42(4):857-907, 1995.

13. L. de Alfaro. Formal Verification of Probabilistic Systems. PhD thesis, Stanford University, 1997.

14. E. A. Emerson and C. Jutla. Tree automata, mu-calculus and determinacy. In Proc. of FOCS, pages 368-377. IEEE, 1991.

15. J. Filar and K. Vrieze. Competitive Markov Decision Processes. Springer, 1997.

16. H. Gimbert and F. Horn. Solving simple stochastic tail games. In Proc. of SODA, pages 847-862, 2010.

17. H. Gimbert, Y. Oualhadj, and S. Paul. Computing optimal strategies for Markov decision processes with parity and positive-average conditions. Technical report, LaBRI, Université de Bordeaux II, Feb 2, 2011. http://hal.archives-ouvertes.fr/docs/00/ 56/18/30/PDF/Gimbert_Oualhadj_Paul_Par_Posavg_MDP.pdf.

18. M. Jurdziński. Deciding the winner in parity games is in UP $\cap$ co-UP. Inf. Process. Lett., 68(3):119-124, 1998.

19. Antonín Kucera and Oldrich Strazovský. On the controller synthesis for finite-state markov decision processes. In FSTTCS, pages 541-552, 2005.

20. J. R. Norris. Markov chains. Cambridge University Press, 1998.

21. A. Pacuk. Hybrid of mean payoff and total payoff. In Talk at the workshop "Games for Design and Verification, St Anne's College Oxford”, September 2010.

22. H. Royden. Real analysis. Prentice Hall, 3rd edition, 12 February 1988.

23. W. Thomas. Languages, automata, and logic. In Handbook of Formal Languages, volume 3, Beyond Words, chapter 7, pages 389-455. Springer, 1997.

24. M.Y. Vardi. Automatic verification of probabilistic concurrent finite-state systems. In FOCS'85. IEEE Computer Society Press, 1985.

25. U. Zwick and M. Paterson. The complexity of mean payoff games on graphs. Theor. Comput. Sci., 158(1\&2):343-359, 1996. 


\section{Appendix}

\section{Details of Section 3}

Proof (of Lemma 2). We show that player 1 has an almost-sure winning strategy in $M$ if and only if player 1 has a winning strategy in the game $G$ (for the same initial credit).

First, we show that if player 1 has an almost-sure winning strategy $\sigma_{M}$ in $M$, then we can construct a winning strategy $\sigma_{G}$ in $G$. We can assume that $\sigma_{M}$ is pure [9, Theorem 5].

To define $\sigma_{G}$, we assign a rank to prefixes of outcomes of $\sigma_{M}$ in $M$ as follows. Prefixes $\rho$ such that $p(\operatorname{Last}(\rho))=0$ get rank 0 . For other prefixes $\rho$ (with $p(\operatorname{Last}(\rho))=$ $1)$, if $\operatorname{Last}(\rho) \in Q_{1}$ is a player-1 state, then $\rho$ gets $\operatorname{rank} 1+\operatorname{rank}(\rho \cdot q)$ where $q$ is such that $\sigma(\rho)(q)=1$; if Last $(\rho) \in Q_{P}$ is a probabilistic state, then $\rho$ gets rank $1+$ $\min \left\{\operatorname{rank}\left(\rho^{\prime}\right) \mid \rho^{\prime}\right.$ is a ranked successor of $\left.\rho\right\}$. Prefixes without ranked successor get no rank. We claim that all prefixes $\rho$ compatible with $\sigma_{M}$ get a (finite) rank. Otherwise, there would exist a non-ranked prefix compatible with $\sigma_{M}$ (thus reachable with positive probability) such that all its extensions are unranked. This would imply that only states with priority 1 are visited from that point on, hence the co-Büchi objective has positive probability, in contradiction with the fact that $\sigma_{M}$ is almost-sure winning for energy Büchi.

We construct the pure strategy $\sigma_{G}$ as follows. Given a play prefix $\rho_{G}$ in $G$, let $h\left(\rho_{G}\right)$ be the sequence obtained from $\rho_{G}$ by deleting all states of the form $(q, d)$ for $q \in Q_{P}$ and $d \in\{\mathrm{L}, \mathrm{R}\}$. Note that $h\left(\rho_{G}\right)$ is a play in $M$. Let $q_{G}=\operatorname{Last}\left(\rho_{G}\right) \in Q_{1}^{\prime}$, we define $\sigma_{G}\left(\rho_{G}\right)$ as follows:

- if $q_{G} \in Q_{1}$, then $\sigma_{G}\left(\rho_{G}\right)=\sigma_{M}\left(h\left(\rho_{G}\right)\right)$;

- if $q_{G}=(q, \mathrm{~L})\left(\right.$ for $\left.q \in Q_{P}\right)$, then $\sigma_{G}\left(\rho_{G}\right)=q^{\prime}$ where $\operatorname{rank}\left(\rho_{G} \cdot q^{\prime}\right)<\operatorname{rank}\left(\rho_{G}\right)$.

Note that for every outcome $\rho_{G}$ of $\sigma_{G}$, the play $h\left(\rho_{G}\right)$ is an outcome of $\sigma_{M}$ in $M$. Towards contradiction, assume that $\sigma_{G}$ is not winning in $G$. Then, there exists an outcome $\rho_{G}$ of $\sigma_{G}$ that violates either:

- the energy condition; then, the energy level drops below 0 after finitely many steps in $\rho_{G}$, and this occurs as well in $h\left(\rho_{G}\right)$ with positive probability in $M$, a contradiction with the fact that $\sigma_{M}$ is almost-sure winning for energy Büchi in $M$.

- or the Büchi condition; then, from some point on in $\rho_{G}$ only priority 1 is visited. This implies that in the gadgets, eventually only $(q, \mathrm{~L})$ states are visited. Then, according to the definition of $\sigma_{G}$, the rank in prefixes of $\rho_{G}$ decreases and eventually reaches rank 0 , that is a state with priority 0 is visited, and we have again a contradiction.

Therefore, $\sigma_{G}$ is a winning strategy in the game $G$.

Second, we show that if player 1 has a winning strategy $\sigma_{G}$ in $G$, then we can construct an almost-sure winning strategy $\sigma_{M}$ in $M$. By the result of [7, Lemma 8] and its proof, we can assume that $\sigma_{G}$ is energy-based memoryless, that is $\sigma_{G}(\rho)=\sigma_{G}\left(\rho^{\prime}\right)$ for all $\rho, \rho^{\prime}$ such that $\operatorname{Last}(\rho)=\operatorname{Last}\left(\rho^{\prime}\right)$ and $\operatorname{EL}(\rho)=\operatorname{EL}\left(\rho^{\prime}\right)$. In particular, if $h(\rho)=$ $h\left(\rho^{\prime}\right)$, then $\sigma_{G}(\rho)=\sigma_{G}\left(\rho^{\prime}\right)$.

We define the strategy $\sigma_{M}$ as follows: for each prefix $\rho_{M}$ in $M$, let $\sigma_{M}\left(\rho_{M}\right)=$ $\sigma_{G}(\rho)$ where $\rho$ is such that $h(\rho)=\rho_{M}$. By the above remark, the strategy $\sigma_{M}$ is 
uniquely and well defined. We also know that $\sigma_{G}$ uses finite memory. Therefore, in $G_{\sigma_{G}}$ all cycles are have nonnegative energy and visit a priority 0 state. Therefore, all cycles in $M_{\sigma_{M}}$ have nonnegative energy; and if there is a reachable closed recurrent set $U$ in $M_{\sigma_{M}}$ that contains only priority 1 states, then in $G_{\sigma_{G}}$ player 2 can fix a strategy to reach the closed recurrent set $U$ (by choosing the successor of probabilistic states using $(\cdot, \mathrm{R})$ states) and in the states of $U$, player 2 always chooses $(\cdot, \mathrm{L})$ states. The (unique) outcome is a play that eventually remains in the closed recurrent set and therefore visits priority 1 states only from some point on, spoiling strategy $\sigma_{G}$, a contradiction. Hence, all closed recurrent sets in $M_{\sigma_{M}}$ contain a priority 0 state and the Büchi objective is satisfied with probability 1 under strategy $\sigma_{M}$.

Proof (of Lemma 3). Consider the construction of $\left\langle M^{\prime}, p^{\prime}, w^{\prime}\right\rangle$ defined before Lemma 3. Let $\operatorname{Win}^{\prime} \subseteq Q \times\{0,2, \ldots, 2 r\}$ be the set of almost-sure winning states in $M^{\prime}$ for the energy Büchi objective and let $\operatorname{Win}=\left\{q \in Q \mid \exists i \cdot(q, 2 i) \in W_{i n}^{\prime}\right\}$ be the projection of $W i n^{\prime}$ on $Q$. We then convert all states in $W$ to absorbing (or sink) states with weight 0 , and then consider almost-sure energy Büchi winning set $Z$ with $W i n$ as the Büchi set (this is almost-sure energy and reachability to Win).

We claim $Z$ is the almost-sure winning set for energy parity in $M$. The proof is as follows. Let $\bar{Z}=Q \backslash Z$. Consider an arbitrary strategy $\sigma$ for player 1 and a starting state $q \in \bar{Z}$. Assume towards contradiction that $\sigma$ is almost-sure winning for energy parity objective. Suppose there is an end-component $U$ such that $U \cap \bar{Z} \neq \emptyset$, that is visited infinitely often with positive probability. Since $\sigma$ is almost-sure winning, we must have that $\min (p(U)$ ) is even (say $2 i$ ) and the energy objective is satisfied. Hence in the copy $2 i$ in $M^{\prime}$ we have that $U$ is almost-sure winning. This means $U \times\{2 i\} \subseteq W^{\prime} i^{\prime}$ and since $U \subseteq Q$ we have $U \subseteq$ Win. But this contradicts that $U \cap \bar{Z} \neq \emptyset$ and Win $\subseteq Z$. It follows that there is no end-component that intersects with $\bar{Z}$ that is visited infinitely often with positive probability. Hence, given $\sigma$, the set $Z$ must be reached with probability 1 . If the energy objective is also ensured with probability 1 by $\sigma$, then the strategy is almostsure winning for energy and reachability to Win (since from $Z$ almost-sure winning for energy and reachability to Win can be ensured). This shows that $q$ would belong to $Z$. This is a contradiction and completes the proof.

Bound for strategies. We construct an almost-sure winning strategy of size at most $2 \cdot(|Z|+1) \cdot W$ as follows. We first partition the set Win as follows: $\operatorname{Win}_{0}$ is the set of states that is winning in copy $0 ; W_{i n}$ is the set of states that is winning in copy 2 and not in copy 0; and so on. For a state $q \in \operatorname{Win}$, let $q \in \operatorname{Win}_{2 i}$, then for the state $q$ we play the almost-sure winning strategy for in copy $2 i$. Since the copies are disjoint, the total memory required for the almost-sure winning strategies is $\sum_{i} 2 \cdot\left|W i n_{2 i}\right| \cdot W=$ $2 \cdot|\operatorname{Win}| \cdot W$. For states $q \in Z \backslash W i n$, we play the almost-sure winning strategy to reach Win ensuring the energy objective. Since for the reachability to Win we can consider states in Win as a single absorbing state, the memory required is at most $2 \cdot(|Z \backslash \operatorname{Win}|+1) \cdot W$. After reaching Win the strategy switches to the almostsure winning strategy from $\mathrm{Win}$. Hence the total memory required by the almost-sure winning strategy is at most $2 \cdot(|Z|+1) \cdot W$.

Algorithm. If we simply apply the algorithm for energy Büchi MDPs on the reduction, then we obtain a $O\left(|E| \cdot d \cdot(d \cdot|Q|)^{5} \cdot W\right)$ algorithm. The improved version is obtain by simply following the steps of the proof. First, for each copy we compute the almost-sure 
winning set for the energy Büchi objective Since each copy is disjoint and in each copy we require $O\left(|E| \cdot|Q|^{5} \cdot W\right)$, the total time required to compute the Win is at most $O\left(|E| \cdot d \cdot|Q|^{5} \cdot W\right)$. Finally the almost-sure energy reachability to Win can be achieved in an additional $O\left(|E| \cdot|Q|^{5} \cdot W\right)$ time. Hence we obtain an $O\left(|E| \cdot d \cdot|Q|^{5} \cdot W\right)$ time algorithm.

\section{Details of Section 4}

Proof (of Lemma 5). The strategy $\sigma^{*}$ for the mean-payoff parity objective is produced by combining two pure memoryless strategies: $\sigma_{m}$ for the expected mean-payoff objective and $\sigma_{Q}$ for the objective of reaching the smallest priority. We present a few properties that we use in the correctness proof of the almost-sure winning strategy.

1. Property 1. Finite-time reach to smallest priority. Observe that under the strategy $\sigma_{Q}$ we obtain a Markov chain such that every closed recurrent set in the Markov chain contains states with the smallest priority, and hence from all states $q$ a state with the smallest priority (which is even) is reached in finite time with probability 1.

2. Property 2. Uniform value. The expected mean-payoff value for all states $q \in Q$ is the same: if we fix the memoryless strategy $\sigma_{u}$ that chooses all successors uniformly at random, then we get a Markov chain as the whole set $Q$ as a closed recurrent set, and hence from all states $q \in Q$ any state $q^{\prime} \in Q$ is reached in finite time with probability 1 , and hence the expected mean-payoff value at $q$ is at least the expected mean-payoff value at $q^{\prime}$. It follows that for all $q, q^{\prime} \in Q$ the expected mean-payoff value at $q$ and $q^{\prime}$ coincide. Let us denote the uniform expected mean-payoff value by $v^{*}$.

3. Property 3. Property of optimal mean-payoff strategy. The strategy $\sigma_{m}$ is a pure memoryless strategy and once it is fixed we obtain a Markov chain. The limit of the average frequency (or Cesaro limit) exists for all states and since $\sigma_{m}$ is optimal it follows that for all states $q \in Q$ we have

$$
\lim _{n \rightarrow \infty} \frac{1}{n} \cdot \sum_{i=1}^{n} \mathbb{E}_{q}^{\sigma_{m}}\left[w\left(\left(\theta_{i}, \theta_{i+1}\right)\right)\right]=v^{*},
$$

where $\theta_{i}$ is the random variable for the $i$-th state of a path. In the resulting Markov chain obtained by fixing $\sigma_{m}$, the expected mean-payoff value for every closed recurrent set must be $v^{*}$; otherwise, if there is a closed recurrent set with expected mean-payoff value less than $v^{*}$, then there must be a closed recurrent set with expected mean-payoff value greater than $v^{*}$ as all states have the uniform value $v^{*}$, but then we obtain a state with expected mean-payoff value greater than $v^{*}$ which contradicts Property 2. Hence from the theory of finite state Markov chains (the almost-sure convergence to the Cesaro limit [20]) we obtain that

$\mathbb{P}_{q}^{\sigma}\left(\left\{\rho \mid \lim _{\ell \rightarrow \infty} \frac{1}{\ell} \cdot \operatorname{EL}(w, \rho(\ell)) \geq v^{*}\right\}\right)=\lim _{\ell \rightarrow \infty} \mathbb{P}_{q}^{\sigma}\left(\left\{\rho \mid \frac{1}{\ell} \cdot \operatorname{EL}(w, \rho(\ell)) \geq v^{*}\right\}\right)=1$.

In the above equality the limit and the probability operators are exchanged using Lesbegue's Dominated Convergence Theorem [22] (as the weights are bounded). Hence for all $\epsilon>0$, there exists $j(\epsilon) \in \mathbb{N}$ such that if $\sigma_{m}$ is played for any $\ell \geq j(\epsilon)$ 
steps then the average of the weights for $\ell$ steps is at least $\epsilon$ within the expected mean-payoff value of the MDP with probability at least $1-\epsilon$, i.e., for all $q \in Q$, for all $\ell \geq j(\epsilon)$ we have

$$
\mathbb{P}_{q}^{\sigma}\left(\left\{\rho \mid \frac{1}{\ell} \cdot \operatorname{EL}(w, \rho(\ell)) \geq v^{*}-\epsilon\right\}\right) \geq 1-\epsilon .
$$

Let $W$ be the maximum absolute value of the weights. The almost-sure strategy $\sigma^{*}$ for mean-payoff parity objective is played in rounds, and the strategy for round $i$ is as follows:

1. Stage 1. First play the strategy $\sigma_{Q}$ till the smallest priority is reached.

2. Stage 2. Let $\epsilon_{i}=1 / i$. If the game was in the first stage in this ( $i$-th round) for $k_{i}$ steps, then play the strategy $\sigma_{m}$ for $\ell_{i}$ steps such that $\ell_{i} \geq \max \left\{j\left(\epsilon_{i}\right), i \cdot k_{i} \cdot W\right\}$. This ensures that the with probability at least $1-\epsilon_{i}$ the average of the weights in round $i$ is at least

$$
\begin{aligned}
\frac{\ell_{i} \cdot\left(v^{*}-\epsilon_{i}\right)-k_{i} \cdot W}{k_{i}+\ell_{i}} & =\frac{\left(\ell_{i}+k_{i}\right) \cdot v^{*}-\ell_{i} \cdot \epsilon_{i}-k_{i} \cdot v^{*}-k_{i} \cdot W}{k_{i}+\ell_{i}} \\
& \geq v^{*}-\frac{\ell_{i} \cdot \epsilon_{i}+k_{i} \cdot v^{*}+k_{i} \cdot W}{\ell_{i}+k_{i}} \\
& \geq v^{*}-\epsilon_{i}-\frac{2 \cdot k_{i} \cdot W}{\ell_{i}+k_{i}} \quad\left(\text { since } v^{*} \leq W\right) \\
& \geq v^{*}-\epsilon-\frac{2 \cdot k_{i} \cdot W}{\ell_{i}} \\
& \geq v^{*}-\epsilon_{i}-\frac{2}{i} \quad\left(\text { since } \ell_{i} \geq i \cdot k \cdot W\right) \\
& =v^{*}-\frac{3}{i} .
\end{aligned}
$$

Then the strategy proceeds to round $i+1$.

The strategy ensures that there are infinitely many rounds (this follows by Property 1 of finite-time reachability to min even priority state). Hence with probability 1 the smallest priority that is visited infinitely often is the smallest priority of the end-component (which is even). This ensures that the parity objective is satisfied with probability 1 . We now argue that the mean-payoff objective is also satisfied with probability 1 . Fix arbitrary $\epsilon>0$ and consider $i$ such that $\frac{3}{i} \leq \epsilon$. For all $j \geq i$, in round $j$, the average weights is at least $v^{*}-\epsilon$ with probability at least $1-\epsilon$. Since mean-payoff objective is independent of finite prefixes, for all $q \in Q$ we have

$$
\mathbb{P}_{q}^{\sigma}\left(\left\{\rho \mid \lim _{\ell \rightarrow \infty} \frac{1}{\ell} \cdot \operatorname{EL}(w, \rho(\ell)) \geq v^{*}-\epsilon\right\}\right) \geq 1-\epsilon .
$$

Since $\epsilon>0$ is arbitrary, letting $\epsilon \rightarrow 0$, we obtain that for all $q \in Q$ we have

$$
\mathbb{P}_{q}^{\sigma}\left(\left\{\rho \mid \lim _{\ell \rightarrow \infty} \frac{1}{\ell} \cdot \operatorname{EL}(w, \rho(\ell)) \geq v^{*}\right\}\right) \geq 1
$$

Hence depending on whether $v^{*} \geq \nu$ or $v^{*}>\nu$ we obtain the desired result. 


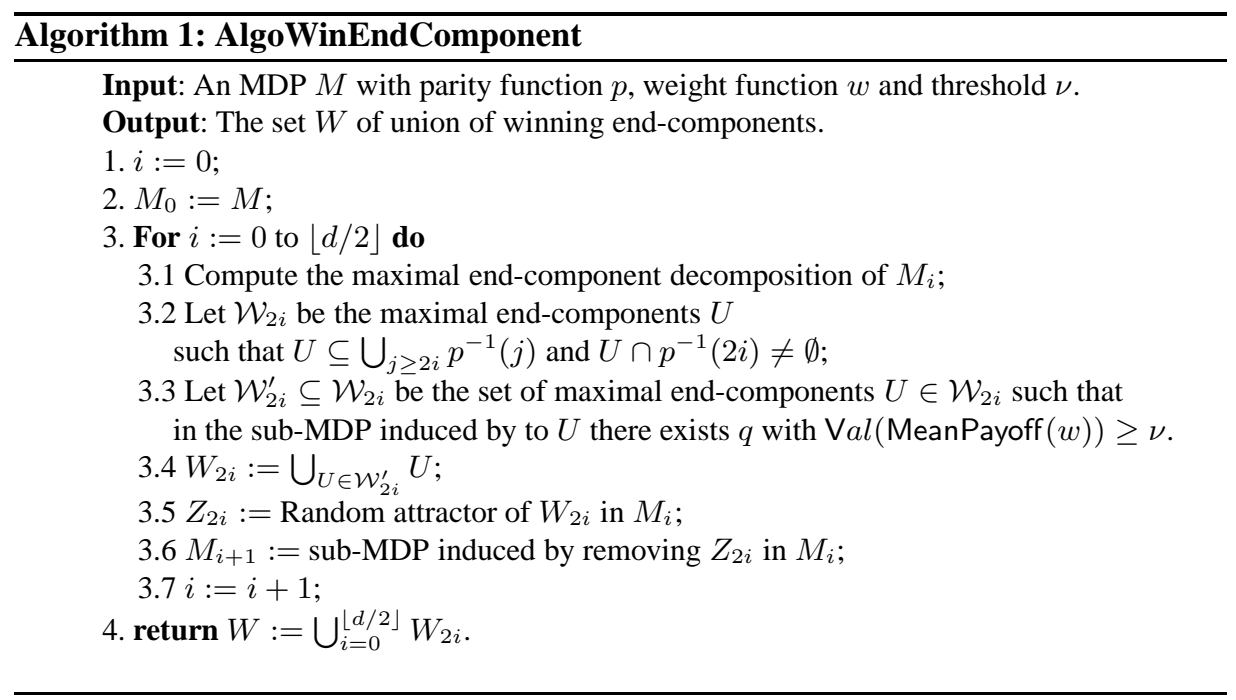

Further details about computing winning end-components for MDPs with meanpayoff parity objectives. We now present some further details about computing the winning end-components with mean-payoff parity objectives. The computation of the winning end-components is done iteratively by computing winning end-components with smallest priority 0 , then winning end-components with smallest priority 2 , and so on. We start with the initial MDP $M_{0}:=M$. In iteration $i$ the remaining MDP is $M_{i}$. We compute the maximal end-component decomposition of $M_{i}$, then consider the maximal-end components $U$ that contains only states with priority at least $2 i$, and at least one state with priority $2 i$. If there is such an end component $U$ where the expected mean-payoff value is at least $\nu$ at some state, then $U$ is included in $W_{2 i}$. The we consider the random attractor (i.e., alternating reachability to $W_{2 i}$ by the random player) to $W_{2 i}$ and the set of random attractor is removed from the MDP for the next iteration. The random attractor to a set $T$ is as follows: $T_{0}:=T$ and for $i \geq 0$ we have $T_{i+1}:=$ $T_{i} \cup\left\{q \in Q_{1} \mid \forall q^{\prime} \in Q .\left(q, q^{\prime}\right) \in E \rightarrow q^{\prime} \in T_{i}\right\} \cup\left\{q \in Q_{P} \mid \exists q^{\prime} \in Q .\left(q, q^{\prime}\right) \in\right.$ $\left.E \wedge q^{\prime} \in T_{i}\right\}$ and the random attractor is $\bigcup_{i \geq 0} T_{i}$. It follows from the results of [10] (see Lemma 2.1 of [10]) that if we consider a set of end-components, and take random attractor to the set, then the maximal end-component decomposition of the remaining MDP remains unaffected. Moreover, the complement of a random attractor in an MDP is always a sub-MDP. The set $W=\bigcup_{i=0}^{\lfloor d / 2\rfloor} W_{2 i}$ is the union of the states of the winning end-components. The formal pseudocode is given as Algorithm 1.

\section{Details of Theorem 3}

We first consider the disjunction of mean-payoff and parity objectives, and then disjunction of energy and parity objectives.

Disjunction of mean-payoff and parity objectives. For the disjunction of meanpayoff and parity objectives in MDPs we consider end-components analysis. An endcomponent is winning if either the parity objective can be ensured almost-surely, or 
the mean-payoff objective can be ensured almost-surely. Since determining almost-sure winning for parity objective and mean-payoff objectives can be done in polynomial time, we can use the algorithm of Section 4 for computing winning end-components and then reachability to winning end-components. Hence disjunction of mean-payoff parity objectives can be solved in polynomial time, and also pure memoryless optimal strategies exist.

Disjunction of energy and parity objectives. The solution of disjunction of energy and parity objectives is achieved using the end-component analysis: an end-component is winning if either the parity objective can be ensured almost-surely or the energy objective can be ensured almost-surely. Whether an end-component is almost-sure winning for parity can be decided in polynomial time, and for energy objectives it is in NP $\cap$ coNP. Hence the winning end-components can be determined in NP $\cap$ coNP. Let $W_{1}$ be the union of the set of winning end-components for almost-sure parity, and let $W_{2}$ be the union of the set of remaining winning end-components (i.e., only almost-sure winning for energy). Finally we need to ensure almost-sure reachability to $W_{1}$ or almost-sure energy reachability to $W_{2}$. Again this can be achieved in NP $\cap$ coNP.

The desired result follows (also see discussion section in [8]). 